\title{
Estimativa de Fluxo Térmico com Variação Temporal Empregando Imagens Termográficas e o Método de Luus-Jaakola
}

\section{Timewise Varying Heat Flux Estimation Employing Infrared Thermography and the Luus- Jaakola Method}

Caroline A. Blaudt ${ }^{1}$, Emerson L. Sanches ${ }^{2}$, Diego C. Knupp ${ }^{3}$, Luiz A. S. Abreu4, Antônio J. Silva Neto ${ }^{5}$

\section{RESUMO}

Este trabalho tem por objetivo a análise da estimativa da variação temporal do fluxo de calor em uma placa termicamente fina a partir de medições de temperatura obtidas via termografia por infravermelho. O problema direto foi formulado considerando-se uma abordagem por parâmetros concentrados (lumped analysis), enquanto o problema inverso foi formulado implicitamente, com a definição de uma função objetivo a ser minimizada. $O$ procedimento de minimização foi então realizado com o algoritmo de Luus-Jaakola. Foram gerados resultados com dados experimentais simulados, com a adição de ruído, para verificação do código. Posteriormente, alguns resultados envolvendo dados experimentais reais foram também empregados. Dos resultados obtidos conclui-se que o modelo empregado descreve satisfatoriamente o problema, dentro das incertezas de cálculo para os parâmetros do modelo e das incertezas dos dados experimentais.

Palavras-chave: Fluxo térmico, Condução de Calor, Problemas Inversos, Regularização

\section{ABSTRACT}

This work addresses the boundary heat flux estimation in thermally thin plates employing temperature measurements obtained via infrared thermography. The direct problem was formulated considering a lumped analysis, while the inverse problem was formulated implicitly, with the definition of an objective function that needs to be minimized. The minimization procedure for this function was then performed with the Luus-Jaakola algorithm. Results were generated with simulated experimental data, with the addition of noise, to verify the code. Once verified, the methodology was applied in a problem involving real experimental data. From the results obtained, it can be concluded that the model adequately simulates the physical problem, within the uncertainties of calculation for the model parameters and the uncertainties regarding experimental data.

Keywords: Heat flux, Heat conduction, Inverse problems, Regularization
${ }^{1}$ Mechanical Engineer,

Universidade do Estado do Rio de Janeiro - UERJ.

E-mail: carolineblaudt@bol.com.br

${ }^{2}$ Mechanical Engineer,

Universidade do Estado do Rio de Janeiro - UERJ.

E-mail:

emerson_Ilpsanches@hotmail.com

${ }^{3}$ D.Sc. Mechanical Enginnering, Universidade do Estado do Rio de Janeiro - UERJ.

E-mail: diegoknupp@iprj.uerj.br

${ }^{4}$ D.Sc. Mechanical Enginnering, Universidade do Estado do Rio de Janeiro - UERJ.

E-mail: luiz.abreu@iprj.uerj.br

${ }^{5}$ Ph.D. Mechanical and

Aerospace Enginnering,

Universidade do Estado do Rio de Janeiro - UERJ.

E-mail: ajsneto@iprj.uerj.br 


\section{INTRODUÇAOO}

Com o advento do uso de tecnologias cada vez menores, principalmente para o desenvolvimento de aparelhos eletrônicos, tais como chips, microchips e nanochips, se faz necessário caracterizar o comportamento do fluxo de calor nesses produtos (HETSRONI et al., 2001). A busca por equipamentos cada vez mais portáteis, leves e de tamanhos reduzidos só se fez possível com o desenvolvimento dos microcomponentes. A energia elétrica utilizada por esses microeletrônicos é parcialmente convertida em energia térmica acarretando um aumento da temperatura do aparelho, que, caso não seja propriamente controlado, pode levar a um mal funcionamento do mesmo ou até danificá-lo. Além do mais, a utilização de dispositivos com tamanho reduzido tem causado pontos não uniformes de superaquecimento, com fluxos de calor muito mais intensos do que a média total no resto do aparelho (BAR-COHEN \& WANG, 2012).

Entender como esse fluxo se comporta mostra-se necessário, pois tais equipamentos são sensíveis a variações bruscas de temperatura e necessitam de estabilidade térmica para assegurar o seu bom funcionamento. Muitas pesquisas são realizadas com o intuito de estimar o comportamento espacial e temporal de fluxos térmicos, visando minimizar e controlar esses danos causados por aquecimentos pontuais (KNUPP \& ABREU, 2016).

Uma importante ferramenta para o estudo do comportamento deste fluxo é a termografia de infravermelho. Trata-se de uma eficiente ferramenta utilizada na medição não intrusiva de temperaturas na superfície de objetos. Este método é atualmente empregado em diversas áreas, tais como inspeção e controle de qualidade na indústria (CAMPOS et al., 2011), determinação de propriedades termofísicas dos materiais (KNUPP et al., 2012), na inspeção preventiva de edifícios para, por exemplo, detectar infiltrações de água (ALTOÉ \& OLIVEIRA FILHO, 2012) e até mesmo no campo da medicina veterinária e produção animal (ROBERTO \& SOUZA, 2014).

Além de ser um método não intrusivo, a termografia por infravermelho oferece novas perspectivas com relação às análises de condução de calor, apresentando amplo número de medidas para a identificação das propriedades termofísicas do material avaliado, tanto no espaço quanto no tempo (KNUPP et al., 2012). Por esta e outras razões, o uso da termografia difundiu-se tanto nos últimos anos.

Este trabalho tem por objetivo estimar o fluxo de calor em uma placa termicamente fina, como geralmente é o caso em circuitos eletrônicos, cujas propriedades são 
conhecidas, a fim de melhor entender como se comporta a distribuição do fluxo térmico neste material. Para alcançar este objetivo, primeiramente é proposto um modelo matemático para o problema de condução de calor em regime permanente, com soluções analíticas e numéricas obtidas diretamente por meio de funções intrínsecas da plataforma Wolfram Mathematica. Para a formulação do problema inverso de estimativa do fluxo térmico é proposta uma abordagem implícita, onde a função objetivo é minimizada utilizando o algoritmo de Luus-Jaakola. De modo a lidar com a dificuldade enfrentada por Blaudt (BLAUDT et al., 2017), no que diz respeito a necessidade de conhecer de forma mais acurada possível o coeficiente de troca térmica entre a placa e o ambiente, este trabalho avança com a realização de experimentos prévios para a estimativa deste importante parâmetro, antes da realização de experimentos para a estimativa do fluxo térmico propriamente dito.

\section{DEFINIÇÃO DO PROBLEMA E FORMULAÇÁO MATEMÁTICA}

O problema físico consiste no processo de transferência de calor por condução e convecção em duas placas com uma resistência delgada, utilizada como aquecedor, entre elas. A resistência, quando submetida a uma tensão elétrica, é responsável por gerar calor por efeito Joule.

Na Figura 1(a) observa-se a representação esquemática da vista lateral do conjunto, com o fluxo de calor aplicado às faces internas das placas, bem como as perdas para o ambiente por convecção, através das superfícies externas. Uma vez que a distribuição do fluxo de calor é simétrica para as duas placas, dado que estas são idênticas, cada uma recebe metade da intensidade do fluxo de calor fornecido pela fita de resistência, e as perdas laterais também são as mesmas para ambas. Dada a simetria do problema, considera-se a análise de apenas uma das placas, como ilustra a Figura 1(b).

Considerando que não há geração interna de energia e que o material é homogêneo, com propriedades invariantes nas direções $x, y$ e $z$, a equação da difusão térmica para a condução de calor no regime transiente pode ser definida como (INCROPERA et al., 2014):

$$
\rho c_{p} \frac{\partial T(x, y, z, t)}{\partial t}=k\left[\frac{\partial^{2} T(x, y, z, t)}{\partial x^{2}}+\frac{\partial^{2} T(x, y, z, t)}{\partial y^{2}}+\frac{\partial^{2} T(x, y, z, t)}{\partial z^{2}}\right]
$$


onde $c_{p}$ é o calor específico, $\rho$ é a massa específica do material e $k$ representa a condutividade térmica.

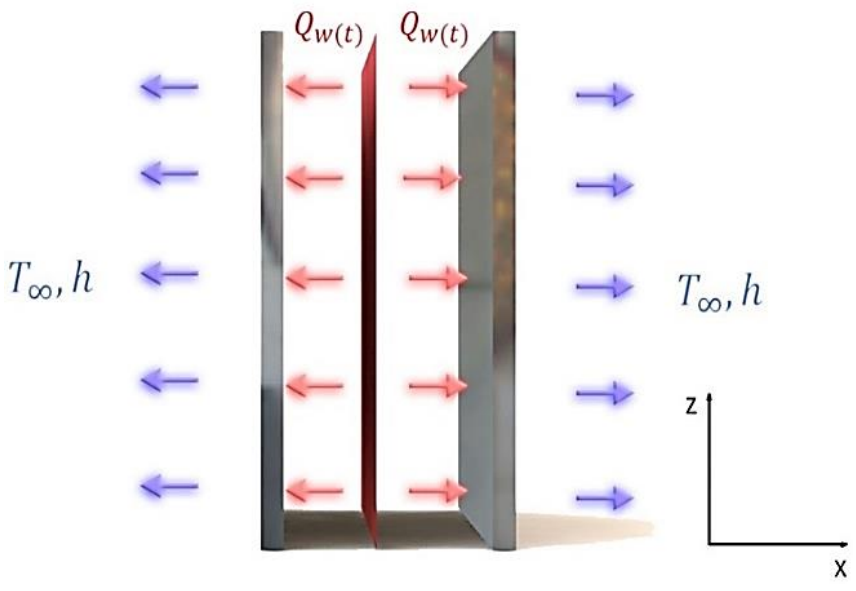

(a)

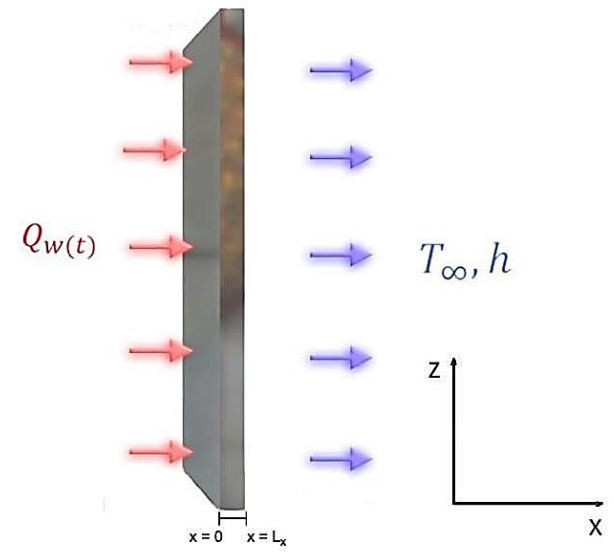

(b)

Figura 1. Representação esquemática do problema físico.

Observando a Figura 1(b) é possível ainda escrever as seguintes condições de contorno e condição inicial para este problema:

$$
\begin{aligned}
& -\left.k \frac{\partial T}{\partial x}\right|_{x=0}=Q_{w}(t) \\
& \left.k \frac{\partial T}{\partial x}\right|_{x=L_{x}}=h_{f}\left(T_{\infty}-T\right) \\
& \left.k \frac{\partial T}{\partial y}\right|_{y=0}=h_{l}\left(T-T_{\infty}\right) \\
& -\left.k \frac{\partial T}{\partial y}\right|_{y=L_{y}}=h_{l}\left(T-T_{\infty}\right) \\
& \left.k \frac{\partial T}{\partial z}\right|_{z=0}=h_{i}\left(T-T_{\infty}\right)
\end{aligned}
$$




$$
\begin{aligned}
& -\left.k \frac{\partial T}{\partial z}\right|_{z=L_{z}}=h_{s}\left(T-T_{\infty}\right) \\
& T(x, y, z, 0)=T_{\infty}
\end{aligned}
$$

onde $L_{x}$ é a espessura da placa, $L_{y}$ é o comprimento, $L_{z}$ a altura, $h$ é o coeficiente de transferência de calor por convecção na face externa, $h_{s}$ o coeficiente de transferência de calor por convecção na parte superior, $h_{i}$ o coeficiente de transferência de calor por convecção na parte inferior da placa, $h_{l}$ o coeficiente de transferência de calor por convecção nas laterais, $Q_{w}(t)$ o fluxo térmico em $x=0$ e $T_{\infty}$ a temperatura do ambiente onde a placa se encontra. Considerando que a placa é fina, usando Lumped Analysys e integrando três vezes nas direções $x, y$ e $z$, é possível obter a seguinte equação que modela o problema físico

$$
\rho c_{p} \frac{d \bar{T}}{d t}=-\frac{h_{f}}{L_{x}}\left(\bar{T}-T_{\infty}\right)+\frac{Q_{w}(t)}{L_{x}}-\frac{2 h_{l}}{L_{y}}\left(\bar{T}-T_{\infty}\right)-\frac{h_{i}}{L_{z}}\left(\bar{T}-T_{\infty}\right)-\frac{h_{s}}{L_{z}}\left(\bar{T}-T_{\infty}\right)
$$

A Eq. (9) possui solução analítica exata, que pode ser prontamente obtida através da rotina DSolve da plataforma de computação simbólico-numérica Wolfram Mathematica. $\mathrm{O}$ objetivo central do presente trabalho consiste em estimar o comportamento temporal do fluxo térmico aplicado nas faces internas das placas, $Q_{w}(t)$, a partir de medições de temperatura obtidas na face externa da placa, i.e. $x=L_{x}$. A formulação e a solução do problema inverso deste respectivo modelo direto são descritas em mais detalhes nas seções subsequentes.

\section{FORMULAÇĀO E SOLUÇÄO DO PROBLEMA INVERSO}

Problemas inversos são problemas mal postos, em que se parte de efeitos observados com a intenção de obter (estimar) causas desconhecidas (CAMPOS VELHO, 2001). Neste trabalho busca-se a solução para o problema inverso de estimativa do fluxo térmico aplicado a uma placa delgada, cujo problema físico foi descrito em detalhes na Seção 2, 
sendo a temperatura na superfície da placa, obtida pelas imagens termográficas, o efeito conhecido e a variação temporal do fluxo térmico a causa que se almeja estimar.

O problema inverso foi formulado implicitamente, com a definição de uma função objetivo. A função objetivo, $S(\mathbf{P})$, empregada neste trabalho é definida como a soma dos resíduos quadrados entre os valores de temperatura calculados com o modelo por meio da solução da Eq. (9), $T_{\text {calc }}$, e os valores de temperatura medidos experimentalmente, $T_{\text {exp }}$ (SILVA NETO \& BECCENERI, 2012):

$$
S(\mathbf{P})=\sum_{i=1}^{N D}\left[\bar{T}_{\exp }\left(t_{i}\right)-\bar{T}_{\text {calc }}\left(t_{i} ; \mathbf{P}\right)\right]^{2}+\gamma \Omega(\mathbf{P})
$$

Neste caso $\boldsymbol{P}$ é o vetor contendo os valores discretos do fluxo de calor em cada instante de tempo onde ele será estimado, a saber

$$
\mathbf{P}=\left\{Q_{w}\left(t_{1}\right), Q_{w}\left(t_{2}\right), Q_{w}\left(t_{3}\right) \ldots Q_{w}\left(t_{N P}\right)\right\}
$$

Além disso, tem-se que $N D$ representa o número de dados experimentais, $N P$ o número de parâmetros sendo estimados e $\Omega(\mathbf{P})$ uma função de regularização ponderada pelo parâmetro de regularização (utilizado para reduzir o efeito dos ruídos experimentais), $\gamma$. A parcela $\gamma \Omega(\mathbf{P})$ é adicionada à função objetivo com a finalidade de se introduzir uma informação a priori sobre o comportamento funcional do fluxo térmico sendo estimado, que neste caso é esperado que tenha comportamento suave em sua maior parte do domínio. $O$ parâmetro $\gamma$ deve ser testado a fim de se obter um melhor ajuste da solução. Valores muito pequenos de $\gamma$ podem levar a um perfil com muitas flutuações, enquanto que valores muito elevados podem acarretar na estimativa de perfis demasiadamente suaves.

A função de regularização empregada no presente trabalho é a de Tikhonov de segunda ordem, expressa por (STEPHANY et al., 2010):

$$
\Omega(\mathbf{P})=\sum_{i=2}^{N P-1}\left(P_{i-1}-2 P_{i}+P_{i+1}\right)^{2}
$$


A fim de se obter uma solução para o problema inverso, a função objetivo apresentada na Eq. (12) precisa ser minimizada. Para tal, emprega-se o algoritmo de Luus-Jaakola. Este algoritmo vem sendo muito utilizado em várias aplicações devido a sua simplicidade de implementação (LUUS, 2001). É uma técnica de busca direta que emprega números aleatórios e reduz o espaço de busca a cada iteração pelo fator de contração ò definido a priori pelo usuário.

Este método consiste em gerar, dentro do domínio de busca, um conjunto de pontos aleatórios a partir de uma solução inicial. Cada ponto testado que apresentar melhor desempenho que o anterior passa a ser classificado como a solução atual, enquanto que o outro ponto é descartado. O fator de contração reduz o domínio de busca e o procedimento continua até que a precisão esperada seja alcançada. O algoritmo de Luus-Jaakola pode ser implementado seguindo o pseudocódigo apresentado na Figura 2 (KNUPP et al., 2016):

Definir um domínio de busca inicial, $\vec{r}^{(0)}$ Escolher o número de loops externos, $n_{\text {out }}$ Escolher o número de loops internos, $n_{\text {int }}$ Escolher o coeficiente de contração, $\varepsilon$ Gerar uma solução inicial, $\vec{x}$

$$
\begin{aligned}
& \text { Para } i=1 \text { até } n_{\text {out }} \\
& \begin{aligned}
\text { Para } j=1 \text { até } n_{\text {int }} \\
\vec{x}^{(j)}=\vec{x}^{*}+\mathbf{R}^{(j)} \vec{r}^{(i-1)} \\
\operatorname{Se~} \mathrm{S}\left(\vec{x}^{(j)}\right)<S(\vec{x}) \\
\vec{x}^{*}=\vec{x}
\end{aligned}
\end{aligned}
$$

Fim se

\section{Fim Para}

$$
\vec{r}^{(i)}=(1-\varepsilon) \vec{r}^{(i-1)}
$$

Fim Para

Figura 2. Pseudocódigo do método de Luus-Jaakola. 


\section{PROCEDIMENTO EXPERIMENTAL}

Para a estimativa dos fluxos térmicos em uma aplicação real, há a necessidade de aquisição de temperaturas experimentalmente. Neste trabalho as temperaturas serão medidas utilizando-se uma técnica não-intrusiva, empregando uma câmera termográfica, disponível no Laboratório Patrícia Oliva Soares de Experimentação e Simulação Numérica em Transferência de Calor e Massa (LEMA-IPRJ/UERJ), que tem seu funcionamento fundamentado no princípio de que qualquer corpo acima da temperatura do zero absoluto emite radiação infravermelha e a intensidade dessa emissão está diretamente relacionada à temperatura do corpo.

Um aparato experimental foi montado para a realização dos experimentos necessários, como ilustra a Figura 3 , com os equipamentos disponíveis no LEMA e destacados na imagem como: (1) Computador; (2) Datalogger Agilent 34972a; (3) Fonte de Corrente Contínua Minipa MPL-1303M; (4) Câmera Termográfica Flir A645sc (640x480 pixels de resolução); (5) Mesa Elevadora ME1 Optron (126x200 mm); (6) Mesa Óptica Optron (600x800 mm); (7) Conjunto de duas placas de alumínio (40x40x2 mm) e uma resistência elétrica e (8) Suporte bilateral para fixação do conjunto.

Além da câmera termográfica, e todos os outros equipamentos necessários ao experimento, outra importante ferramenta é o software Flir ResearchIR, em destaque no computador (item 1) da bancada experimental (Fig. 3), no qual são realizadas as análises, manipulações e as importações finais, numéricas e gráficas.

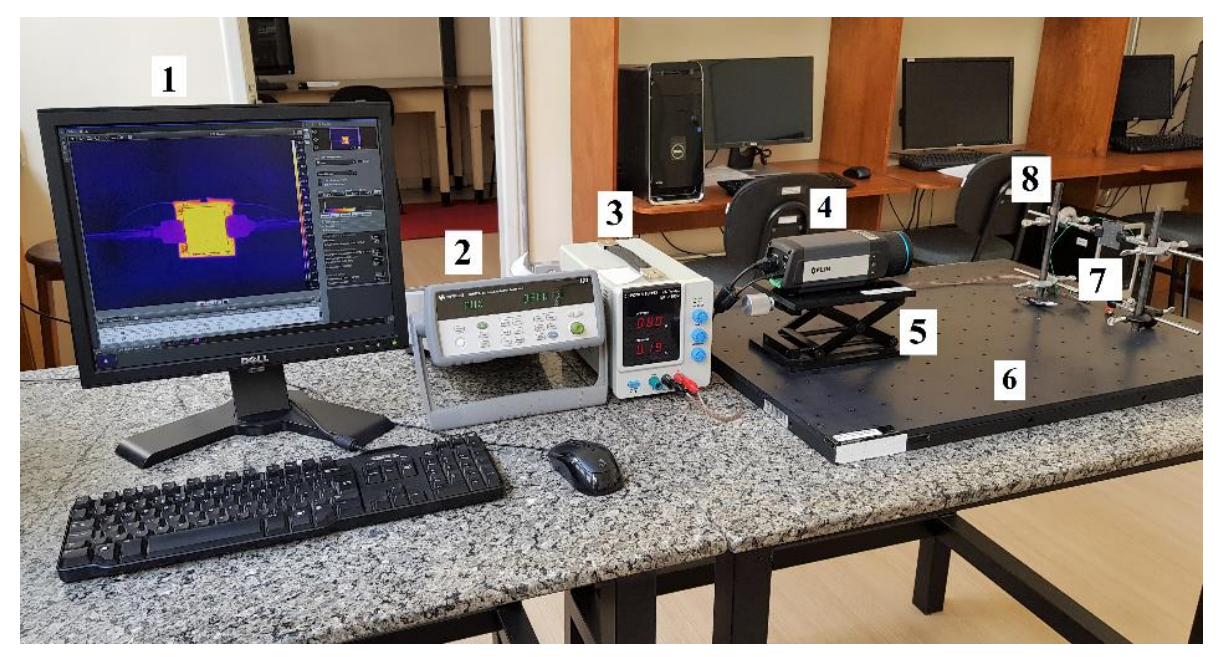

Figura 3. Aparato Experimental. 
A termografia por infravermelho é uma ferramenta para medição de temperatura amplamente empregada em aplicações que exijam nenhuma interferência, sendo este, assim, um método não intrusivo. Para o problema aqui abordado, utiliza-se este método para a obtenção da temperatura média da amostra, submetida ao aquecimento espacialmente uniforme, ao longo do tempo. Cabe ressaltar que os experimentos são realizados com um conjunto de duas placas e uma resistência, em um formato tipo "sanduíche", e a análise é feita em apenas uma das amostras, garantindo, assim, a equivalência com o modelo descrito. $O$ calor dissipado por efeito Joule pela resistência que está entre as placas pode ser então calculado, para fins de validação, a partir do conhecimento da corrente elétrica que é aplicada no circuito.

\section{RESULTADOS E DISCUSSÃO}

Como mencionado na seção anterior, para fins de validação da metodologia proposta, o valor esperado para o fluxo de calor por efeito Joule gerado pela resistência, $Q_{e s p}$, pode ser calculado pela seguinte equação, desprezando-se a inércia térmica do aquecedor (a resistência é fina e sua massa é muito pequena em comparação com as placas):

$$
Q_{e s p}=\frac{R i^{2}}{\left(2 L_{z} L_{y}\right)}
$$

onde $R$ é a resistência do aquecedor, que neste caso é $R=39,9 \Omega$, e $i$ é a corrente aplicada, no valor de $i=0,21 \mathrm{~A}$, resultando em $Q_{e s p} \cong 549,9 \mathrm{~W} / \mathrm{m}^{2}$. Este valor calculado para o fluxo de calor mantém-se constante de $t=300 \mathrm{~s}$ à $t=1500 \mathrm{~s}$, período de tempo em que a resistência está ligada, e considera-se fluxo nulo para outros valores de $t$, estando a resistência desligada, portanto $Q_{e s p}=0$. O valor calculado na Eq. (13) foi utilizado para fins de comparação com a solução do problema inverso obtida neste trabalho.

As propriedades das placas de alumínio, utilizadas como caso teste, foram consideradas conhecidas, sendo $\rho=2703 \mathrm{~kg} / \mathrm{m}^{3}, \quad c_{p}=903 \mathrm{~J} / \mathrm{kgK}$ e $T_{\infty}=25^{\circ} \mathrm{C}$, assim como os parâmetros geométricos, $L_{y}=40 \mathrm{~mm}, L_{z}=40 \mathrm{~mm}$ e $L_{x}=2 \mathrm{~mm}$. O número de dados experimentais considerado foi $N D=1790$ e o número de parâmetros sendo estimados $N P=31$. 
Um parâmetro crucial para o modelo descrito na Seção 2 deste trabalho é o coeficiente de troca térmica por convecção. Por simplicidade, consideremos que todos os coeficientes (em todas as superfícies da placa) são constantes e iguais: $h_{f}=h_{l}=h_{i}=h_{s}=h$. Para estimar o valor de $h$, utilizou-se a solução em regime permanente com fluxo térmico uniforme e conhecido $Q_{\max }$ do modelo descrito pela Eq. (9). Neste caso, a seguinte expressão pode ser obtida:

$$
h=\frac{Q_{\text {Max }}}{T_{m}^{\exp }-T_{\infty}} \frac{L_{y} L_{z}}{\left(L_{y} L_{z}+2 L_{x} L_{z}+2 L_{x} L_{y}\right)}
$$

onde $T_{m}^{\exp }$ é a temperatura média da superfície da placa, medida experimentalmente, quando a placa (com um fluxo $Q_{\text {Max }}$ aplicado) entra em regime permanente.

Com o modelo descrito pela Eq. (14), realizou-se três experimentos independentes, com a amostra de alumínio submetida a três valores de $Q_{\operatorname{Max}}$, para aquisição, via termografia por infravermelho, das temperaturas $T_{m}^{\exp }$ nos respectivos regimes permanentes. Por fim, calculados os valores de $h$ referentes a cada um dos testes, obtevese, através de uma média aritmética, um coeficiente de troca térmica final $h \cong 20,6 \mathrm{~W} / \mathrm{m}^{2} \mathrm{~K}$, que é adotado como conhecido em todos os casos. Além disso, dada a incerteza do valor real do coeficiente de troca térmica, realizou-se a solução do problema inverso também para valores $20 \%$ menores e maiores que esta estimativa, resultando em $h_{-} \cong 16,48 \mathrm{~W} / \mathrm{m}^{2} \mathrm{~K}$ e $h_{+} \cong 24,72 \mathrm{~W} / \mathrm{m}^{2} \mathrm{~K}$, respectivamente.

A estimativa do fluxo de calor com variação temporal foi realizada utilizando os seguintes parâmetros no algoritmo de Luus-Jaakola: o número de loops externos $n_{\text {out }}$, 0 número de loops internos $n_{\text {int }}$, o coeficiente de contração $\varepsilon$ e o parâmetro de regularização $\gamma$.

Tais parâmetros foram previamente testados via dados experimentais simulados e mostraram satisfazer o conjunto de dados experimentais reais, sendo estes $n_{\text {out }}=400$, $n_{\text {int }}=400, \varepsilon=0,01315$ e $\gamma=6 \times 10^{-5} \mathrm{~m}^{4 \circ} \mathrm{C}^{2} / W^{2}$. 
Na Figura 4 é possível observar o resultado obtido para a estimativa do fluxo térmico, fixando-se o coeficiente de troca térmica nos três valores distintos mencionados acima: $h_{-} \cong 16,48 \mathrm{~W} / \mathrm{m}^{2} \mathrm{~K}, h \cong 20,6 \mathrm{~W} / \mathrm{m}^{2} \mathrm{~K}$ e $h_{+} \cong 24,72 \mathrm{~W} / \mathrm{m}^{2} \mathrm{~K}$.

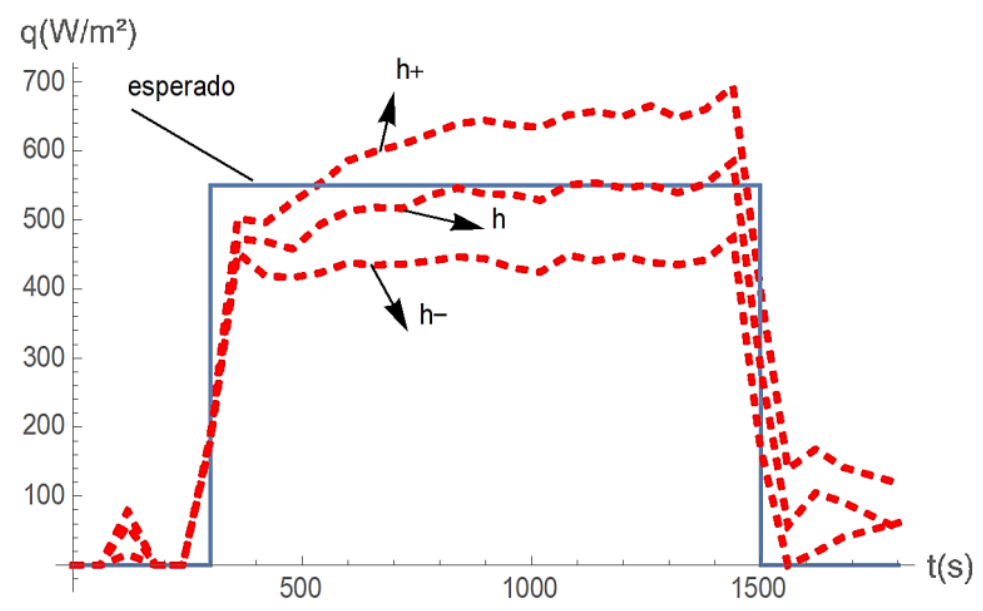

Figura 4. Estimativa do fluxo de calor (linhas tracejadas) em comparação com o comportamento transiente esperado do fluxo (linha sólida).

É possível observar que as estimativas obtidas são capazes de recuperar satisfatoriamente o fluxo esperado, ressaltando-se uma discrepância maior na primeira transição, quando o aquecedor é ligado, onde as estimativas tendem a ser ligeiramente menores que os valores esperados, e comportamento similar na segunda transição, quando o aquecedor é desligado, onde as estimativas tendem a ser ligeiramente maiores que os valores esperados. Este fato pode ser explicado pela inércia térmica do aquecedor, que é desconsiderada na representação do fluxo esperado, de modo que, na realidade, o fluxo não atinge o valor máximo instantaneamente quando o aquecedor é ligado, e nem vai a zero instantaneamente quando o mesmo é desligado.

Outra observação importante é que o valor estimado para o coeficiente de troca térmica em experimentos prévios, e fixado na solução do problema inverso para a determinação do fluxo, é bastante adequado, o que pode ser confirmado pela boa estimativa do fluxo ao se utilizar o valor médio do coeficiente de troca térmica, $h$, assim como a obtenção de um envelope relativamente estreito para o fluxo térmico ao considerar variações de mais e menos $20 \%$ do valor médio do coeficiente de troca térmica, $h_{+}$e $h_{-}$, respectivamente. 
A Figura 5 apresenta a curva de resíduos, que expressa os resíduos entre as temperaturas calculadas e experimentais para a estimativa do fluxo obtida fixando-se o coeficiente de troca térmica no valor médio, $h$.

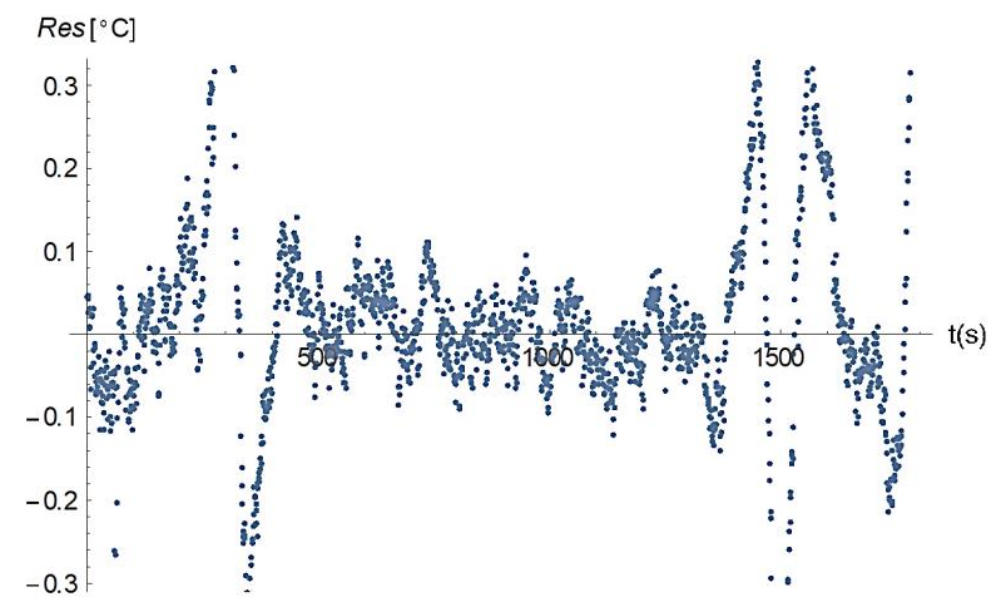

Figura 5. Resíduos entre as temperaturas calculadas com o fluxo estimado e as medições experimentais.

É possível observar que os resíduos apresentam pequenas magnitudes, estando em torno de $0,1^{\circ} \mathrm{C}$ na maior parte dos pontos. Nos pontos de transição (momentos em que o aquecedor é ligado e desligado), os resíduos apresentam valores de maior magnitude, mas que ainda podem ser considerados baixos, da ordem de $0,3^{\circ} \mathrm{C}$.

Assim como para as diferenças observadas nas curvas da Figura 4, a correlação de maiores resíduos com os pontos de transição abrupta do fluxo pode ser explicada, também, pela inércia térmica do aquecedor, que é desprezada no modelo empregado, resultando em desvios ligeiramente maiores entre as temperaturas calculadas e os dados experimentais.

Por fim, a comparação entre a temperatura calculada e a temperatura experimental é apresentada na Figura 6 abaixo, na qual é possível observar que as duas curvas estão satisfatoriamente sobrepostas dentro da escala gráfica considerada. Pequenos desvios são observados apenas nas zonas de transição, quando a resistência é ligada e desligada, como já evidenciado nos resultados anteriores. 


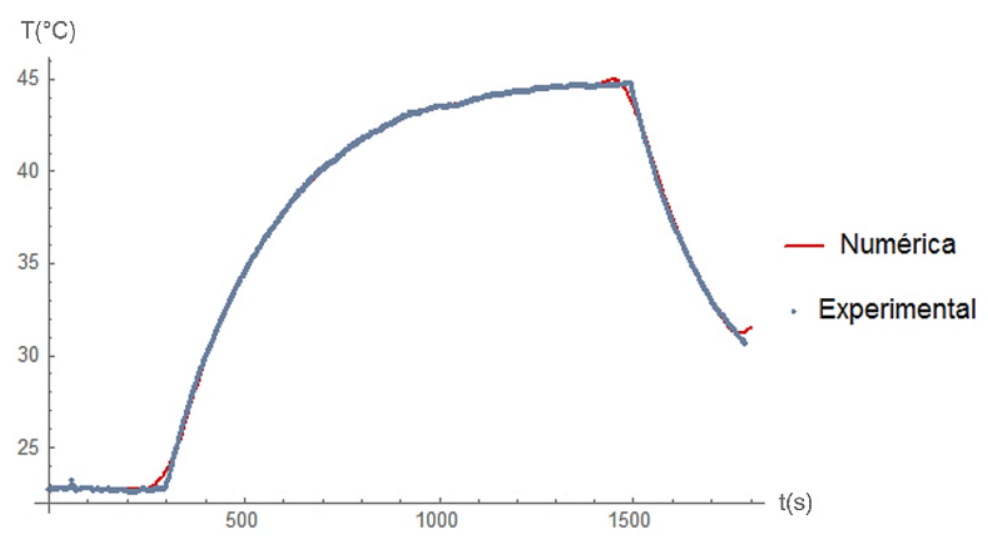

Figura 6. Evolução temporal da temperatura $T(t)$ calculada com o fluxo estimado em comparação com as medições experimentais.

\section{CONCLUSÓES}

Neste trabalho foi desenvolvido um modelo computacional para estimar o fluxo de calor transiente em uma placa termicamente fina, a partir de medidas experimentais de temperatura obtidas utilizando termografia por infravermelho.

O problema inverso foi resolvido a partir da definição de uma função objetivo com regularização a ser minimizada pelo algoritmo de Luus-Jaakola. A estimativa do fluxo térmico foi realizada assumindo-se conhecido o valor do coeficiente de troca térmica entre a superfície da placa e ambiente, tendo sido este previamente obtido através de experimentos independentes.

Os resultados obtidos se mostraram adequados dentro do limite de erros oriundos do procedimento experimental e simplificações empregadas no modelo. A regularização empregada e o método de Luus-Jaakola como método de minimização da função objetivo se mostraram eficientes para o problema proposto, podendo ser futuramente ampliada a outras aplicações

\section{AGRADECIMENTOS}

Os autores agradecem ao apoio das agências de fomento CAPES, CNPq e FAPERJ.

\section{REFERÉNCIAS}

ALTOÉ, L.; OLIVEIRA FILHO, D. Termografia infravermelha aplicada à inspeção de edifícios. Acta Tecnológica. v. 7, n. 1, p. 55-59, 2012.

BAR-COHEN, A.; WANG, P. Thermal management of on-chip hot spot. Journal of Heat Transfer. v. 134, n. 5, p. 1-11, mai. 2012. 
BLAUDT, C.A.; KNUPP, D.C.; ABREU, L.A.S.; SILVA NETO, A.J. Estimativa de Fluxo Térmico empregando Imagens Termográficas e o Método de Luus-Jaakola. XX Encontro Nacional de Modelagem Computacional e VIII Encontro de Ciência e Tecnologia de Materiais, Nova Friburgo, 2017.

CAMPOS, R. B.; CARVALHO, F. C.; FILHO, P. C. M. L.; BRITO, J. N. Inspeção Termográfica de Equipamentos Industriais - Casos de Sucesso. In: XI Congresso Nacional De Engenharia Mecânica, Metalúrgica E Industrial, Porto Alegre, 2011.

CAMPOS VELHO, H. F. Problemas Inversos: Conceitos Básicos e Aplicações. In: IV Encontro De Modelagem Computacional, Instituto Politécnico do Estado do Rio de Janeiro, Nova Friburgo, 2001.

HETSRONI, G.; MOSYAK, A.; SEGAL, Z. Nonuniform temperature distribution in electronic devices cooled by flow in parallel microchannels. IEEE Transactions on Components and Packaging Technologies, v. 24, n. 1, p. 16-23, mar. 2001.

INCROPERA, F. P.; DEWITT, D. P; BERGMAN, T. L.; LAVINE, A. S. Fundamentos de Transferência de Calor e de Massa. 7.ed. Rio de Janeiro: LTC, 2014.

KNUPP, D.C.; ABREU, L. A. S. Explicit boundary heat flux reconstruction employing temperature measurements regularized via truncated eigenfunction expansions. International Communications in Heat and Mass Transfer, v. 78, p. 241-252, 2016.

KNUPP, D. C., NAVEIRA-COTTA, C. P., AYRES, J. V. C., ORLANDE, H. B. O., COTTA, R. $M$. Space-Variable Thermophysical Properties Identification in Nanocomposites via Integral Transforms, Bayesian Inference and Infrared Thermography. Inverse Problems in Science and Engineering, vol. 20 (5), pp. 609-637, 2012.

KNUPP, D. C.; SACCO, W. F.; SILVA NETO, A. J. Método de Luus-Jaakola. In: SILVA NETO, A. J.; BECCENERI, J. C.; CAMPOS VELHO, H. F. Inteligência Computacional Aplicada a Problemas Inversos em Transferência Radiativa. Rio de Janeiro: EdUERJ, 2016. p. 203-211.

LUUS, R. Use of Luus-Jaakola optimization procedure for singular optimal control problems. Nonlinear Analysis: Theory, Methods \& Applications, v. 47, n. 8, p. 5647-5658, ago. 2001

ROBERTO, J. V. B.; DE SOUZA, B. B. Utilização da termografia de infravermelho na medicina veterinária e na produção animal. Journal of Animal Behavior and Biometeorology, v. 2, n.3, p. 73-84, abr. 2014.

SILVA NETO, A. J.; BECCENERI, J. C. Técnicas de Inteligência Computacional Inspiradas na Natureza-Aplicação em Problemas Inversos em Transferência Radiativa. Sociedade Brasileira de Matemática aplicada e Computacional (SBMAC), 2012, $148 \mathrm{p}$.

STEPHANY, S.; BECCENERI, J.C.; SOUTO, R.P.; CAMPOS VELHO, H.F.; SILVA NETO, A. A pre-regularization scheme for the reconstruction of a spatial dependent scattering albedo using a hybrid ant colony optimization implementation. Applied Mathematical Modelling, v. 34, n. 3, p.561-572, mar. 2010. 